\title{
Statistical aspects of nuclear coupling to continuum
}

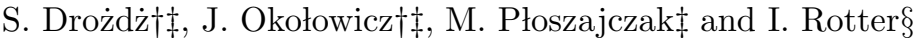 \\ $\dagger$ Institute of Nuclear Physics, Radzikowskiego 152, PL - 31342 Kraków, Poland \\ $\ddagger$ Grand Accélérateur National d'Ions Lourds, CEA/DSM - CNRS/IN2P3, BP 5027, F-14076 Caen Cedex 05, France \\ $\S$ Max-Planck-Institut für Physik komplexer Systeme, D-01187 Dresden, Germany
}

\begin{abstract}
Various global characteristics of the coupling between the bound and scattering states are explicitly studied based on realistic Shell Model Embedded in the Continuum. In particular, such characteristics are related to those of the scattering ensemble. It is found that in the region of higher density of states the coupling to continuum is largely consistent with the statistical model. However, assumption of channel equivalence in the statistical model is, in general, violated.
\end{abstract}

Relating properties of nuclei to the ensembles of random matrices [i] is of great interest. A potential agreement reflects those aspects that are generic and thus do not depend on the detailed form of the Hamiltonian matrix, while deviations identify certain system-specific, non-random properties of the system. On the level of bound states the related issues are quite well explored and documented in the literature [2,3]. In many cases, however, the nuclear states are embedded in the continuum and the system should be considered as an open quantum system. Applicability of the related scattering ensemble of non-Hermitian random matrices [4, 5] has however never been verified by an explicit calculation due to serious difficulties that such an explicit treatment of all elements needed involves. These include a proper handling of multi-exciton internal excitations, an appropriate scattering asymptotics of the states in continuum and a consistent and realistic coupling among the two. The recently developed [5] advanced computational scheme termed the Shell Model Embedded in the Continuum (SMEC) successfully incorporates such elements and will be used below to study conditions under which the statistical description of the continuum coupling applies.

Constructing the full SMEC solution consists of three steps. In the first step, one solves the many-body problem in the subspace $Q$ of (quasi-)bound states. For that one solves the multiconfigurational Shell Model (SM) problem : $H_{Q Q} \Phi_{i}=E_{i} \Phi_{i}$, where $H_{Q Q} \equiv Q H Q$ is the SM effective Hamiltonian which is appropriate for the SM configuration space used. For the continuum part (subspace $P$ ), one solves the coupled channel equations :

$$
\left(E^{(+)}-H_{P P}\right) \xi_{E}^{c(+)} \equiv \sum_{c^{\prime}}\left(E^{(+)}-H_{c c^{\prime}}\right) \xi_{E}^{c^{\prime}(+)}=0
$$

where index $c$ denotes different channels and $H_{P P} \equiv$ $P H P$. The superscript $(+)$ means that boundary conditions for incoming wave in the channel $c$ and outgoing scattering waves in all channels are used. The channel states are defined by coupling of one nucleon in the scattering continuum to the many-body SM state in $(N-1)$ nucleus. Finally one solves the system of inhomogeneous coupled channel equations :

$$
\left(E^{(+)}-H_{P P}\right) \omega_{i}^{(+)}=H_{P Q} \Phi_{i} \equiv w_{i}
$$

with the source term $w_{i}$ which is primarily given by the structure of $N$ - particle SM wave function $\Phi_{i}$ and which couples the wave function of $N$-nucleon localized states with $(N-1)$-nucleon localized states plus one nucleon in the continuum $[6]$. These equations define functions $\omega_{i}^{(+)}$, which describe the decay of quasi-bound state $\Phi_{i}$ in the continuum.

The resulting full solution of SMEC equations is then expressed as [6:7] :

$$
\Psi_{E}^{c}=\xi_{E}^{c}+\sum_{i, j}\left(\Phi_{i}+\omega_{i}\right) \frac{1}{E-H_{Q Q}^{e f f}}\left\langle\Phi_{j}\left|H_{Q P}\right| \xi_{E}^{c}\right\rangle,
$$

where

$$
H_{Q Q}^{e f f}=H_{Q Q}+H_{Q P} G_{P}^{(+)} H_{P Q} \equiv H_{Q Q}+W
$$

defines the effective Hamiltonian acting in the space of quasibound states. Its first term reflects the original direct mixing while the second term originates from the mixing via the coupling to the continuum. $G_{P}^{(+)}$is the Green function for the single particle (s.p.) motion in the $P$ subspace. This external mixing is thus energy dependent and consists of the principal value integral and the residuum :

$$
\begin{aligned}
W_{i j}(E) & =\sum_{c=1}^{\Lambda} \int_{\epsilon_{c}}^{\infty} d E^{\prime} \frac{\left\langle\Phi_{j}\left|H_{Q P}\right| \xi_{E}^{c}\right\rangle\left\langle\xi_{E}^{c}\left|H_{P Q}\right| \Phi_{i}\right\rangle}{E-E^{\prime}} \\
& -i \pi \sum_{c=1}^{\Lambda}\left\langle\Phi_{j}\left|H_{Q P}\right| \xi_{E}^{c}\right\rangle\left\langle\xi_{E}^{c}\left|H_{P Q}\right| \Phi_{i}\right\rangle .
\end{aligned}
$$

These two terms prescribe the structure of the real $W^{R}$ (Hermitian) and imaginary $W^{I}$ (anti-Hermitian) parts of $W$, respectively. The dyadic product form of the second term allows to express it as

$$
W^{I}=-\frac{i}{2} \mathbf{V} \mathbf{V}^{T}
$$

where the $M \times \Lambda$ matrix $\mathbf{V} \equiv\left\{V_{i}^{c}\right\}$ denotes the amplitudes connecting the state $\Phi_{i}(i=1, \ldots, M)$ to the 
reaction channel $c(c=1, \ldots, \Lambda)$ [8]. This form of $W^{I}$ constitutes a starting point towards statistical description of the related effects. In the latter case one assumes that the internal dynamics is governed by the Gaussian orthogonal ensemble (GOE) of random matrices. Relation of this assumption to the classical chaotic scattering can also be traced [9]. The orthogonal invariance arguments then imply that the amplitudes $V_{i}^{c}$ can be assumed to be Gaussian distributed and the channels independent [4. Assuming, as consistent with the statistical ensemble, the equivalence of the channels one then arrives at the following distribution of the off-diagonal matrix elements of $W^{I}$ for $\Lambda$ open channels :

$$
\mathcal{P}_{\Lambda}\left(W_{i j}^{I}\right)=\frac{\left|W_{i j}^{I}\right|^{(\Lambda-1) / 2} K_{(\Lambda-1) / 2}\left(\left|W_{i j}^{I}\right|\right)}{\Gamma(\Lambda / 2) \sqrt{\pi} 2^{(\Lambda-1) / 2}},
$$

with $\left\langle\left(W_{i j}^{I}\right)^{2}\right\rangle=\Lambda . K_{\lambda}$ denotes here the modified Bessel function.

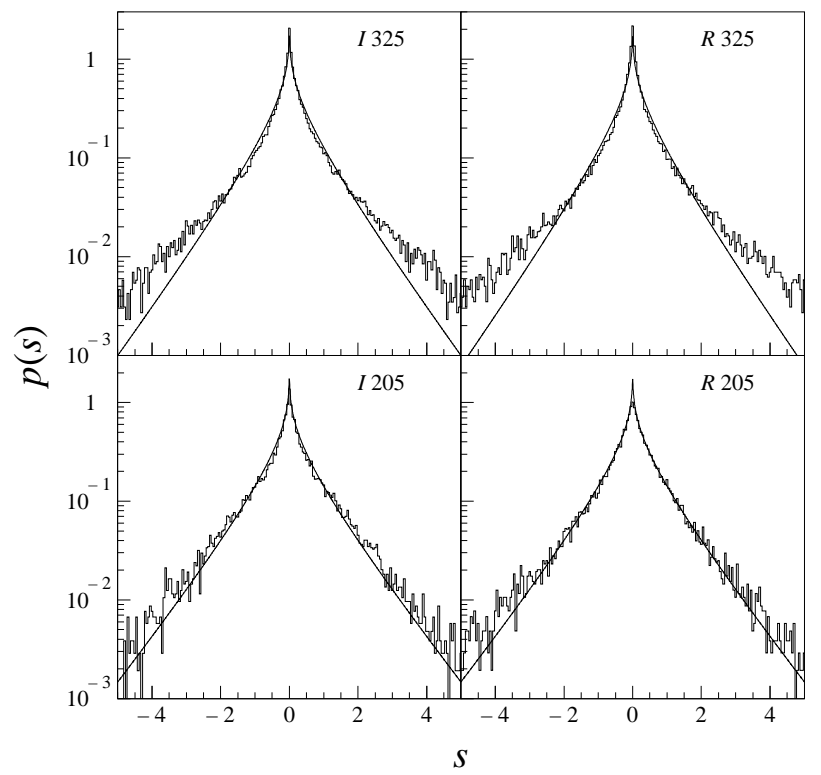

FIG. 1. Typical projections of distribution of matrix $W$ elements coupling to the one channel continuum in the $\mathrm{SM}$ basis of $J^{\pi}=0^{+}, T=0$ states in ${ }^{24} \mathrm{Mg}$ (histograms). The projections on imaginary (left) and real axis (right) are normalized and plotted versus normalized variable $s=\left(W_{i j}^{X}-\left\langle W_{i j}^{X}\right\rangle\right) / \sigma_{X}$, where $\sigma_{X}=\left\langle W_{i j}^{X^{2}}\right\rangle^{1 / 2}$, and $X=I, R$ denotes imaginary and real parts respectively. In the upper parts all 325 states were taken into account, while in the lower parts only 205 states in the middle of the spectrum were included. The full curves represent $\Lambda=1$ distribution (Eq. (日))

The physics to be addressed below by making use of the above formalism is that of a nucleus decaying by the emission of one nucleon. As an example, ${ }^{24} \mathrm{Mg}$ is taken with the inner core of ${ }^{16} \mathrm{O}$ and the phenomenological $s d$-shell interaction among valence nucleons [10]. For the coupling between bound and scattering states a combination of Wigner and Bartlett forces is used, with the spin-exchange parameter $\beta=0.05$ and the overall strength coupling $V_{12}^{(0)}=650 \mathrm{MeV} \cdot \mathrm{fm}^{3}$ [6]. The radial s.p. wave functions in the $Q$ subspace and the scattering wave functions in $P$ subspace are generated from the average potential of the Woods-Saxon type [6].

In the above SM space, the ${ }^{24} \mathrm{Mg}$ nucleus has $325 J^{\pi}=$ $0^{+}, T=0$ states. Depending on the particle emission threshold, these states can couple to a number of open channels. Such channels correspond to excited states in the neighboring $N-1$ nucleus.

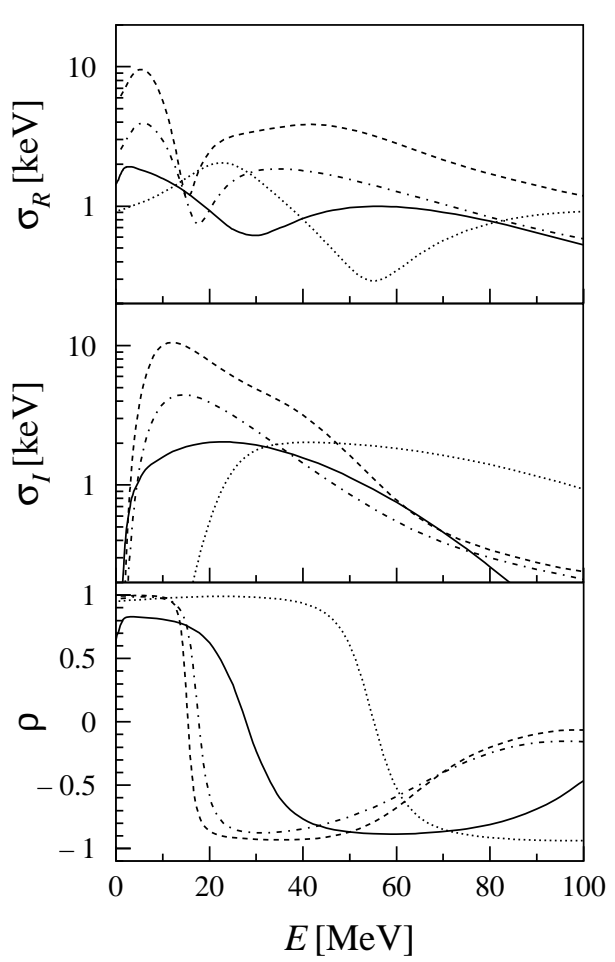

FIG. 2. Variance of real $\left(\sigma_{R}\right)$ and imaginary $\left(\sigma_{I}\right)$ parts of matrix elements $W_{i j}$ for one open channel and correlation coefficient $\rho=\left(\left\langle W_{i j}^{R} W_{i j}^{I}\right\rangle-\left\langle W_{i j}^{R}\right\rangle\left\langle W_{i j}^{I}\right\rangle\right) /\left(\sigma_{R} \sigma_{I}\right)$ between them. Different line styles correspond to different daughter nucleus spins: 1/2 (full line), 3/2 (dashed line), 5/2 (dot-dash) and $7 / 2$ (dots). All these quantities are shown as a function of energy of the particle in the continuum.

When testing validity of the statistical model it is instructive to begin with one open channel and to compare the distribution of the corresponding matrix elements with the formula (7) for $\Lambda=1$. In the example shown in Fig. 1, the open channel corresponds to spin $1 / 2$ and its energy to about the middle of the spectrum. Both the imaginary (left) and real (right) parts of $W$ are displayed. The upper part of Fig. 1 involves all 325 $J^{\pi}=0^{+}, T=0$ states of ${ }^{24} \mathrm{Mg}$. Clearly, there are too many large and also too many small matrix elements as compared to the statistical distribution (solid line) with $\Lambda=1$. This may originate from the fact that many 
states in the $Q$ space are localized stronger than allowed by the GOE. It is actually natural to suspect that this may apply to the states close to both edges of the spectrum. Indeed, by discarding 60 states on both ends of the spectrum (205 remain), the picture changes significantly as illustrated in the lower part of Fig. 11. In this case the statistical distribution provides a good representation, interestingly, also for the real part although applicability of the formula (7) is not directly justifiable as for the imaginary part. Similar behavior is found for majority of channels except for a limited number of them located at the edges of the spectrum. Hence, the assumption about the Gaussian distribution of amplitudes $V_{i}^{c}$ is well fulfilled in a generic situation.

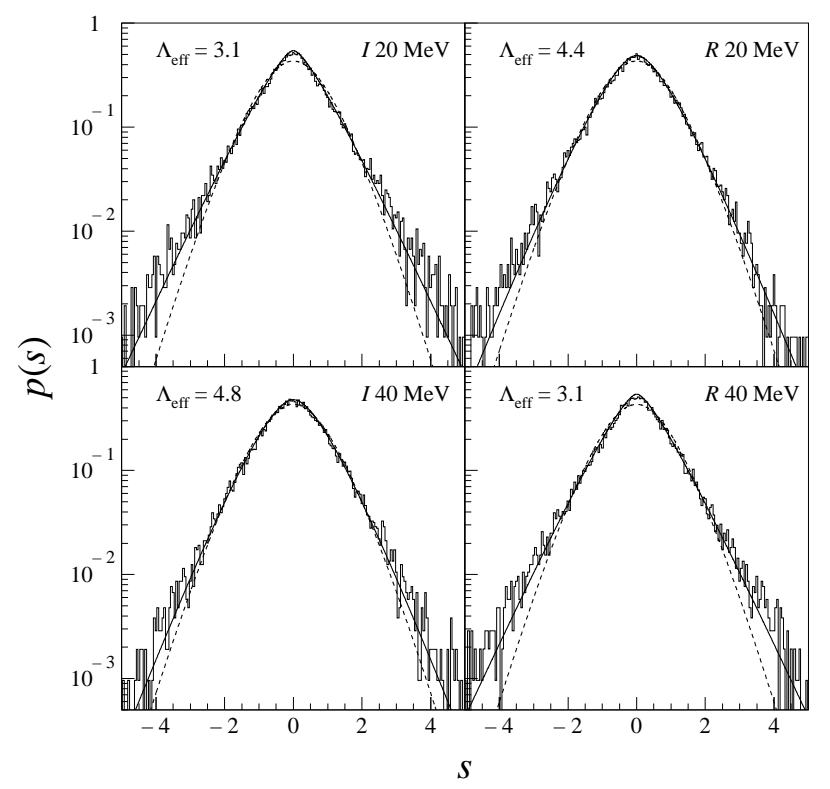

FIG. 3. The same as lower part of Fig. 11 but for 10 channels of spins ranging from $1 / 2$ to $7 / 2$ and two energies of the particle in the continuum (depicted in the figure). The full curves represent $\mathcal{P}_{\Lambda_{\text {eff }}}$ fits with $\Lambda_{\text {eff }}$ indicated while the dashed curves correspond to distribution with $\Lambda=10$.

As for the equivalence of channels, the conditions are expected to be more intricate, especially when different channel quantum numbers are involved, because the effective coupling strength depends on those quantum numbers. In addition, such a coupling strength depends also on energy $E$ of the particle in continuum so the proportions among the channels may vary with $E$. This is illustrated in Fig. 2 which shows the energy dependence of the standard deviations of distributions (as in Fig. 11) of relevant matrix elements for several different channel spin values, both for real and imaginary part of $W$, and their correlation coefficient. It should to be noted however that within a given spin the differences are much smaller. Instead of trying to identify (with the help of Fig. 2) a sequence of $\Lambda$ approximately equivalent channels and to verify the resulting distribution of matrix elements of $W$ against formula (7) we find it more informative to make a random selection of such channels. An example for $\Lambda=10$ and two different energies $(E=20$ and $40 \mathrm{MeV}$ ) of the particle in the continuum is shown in Fig. 3. Among these 10 randomly selected channels, two correspond to spin $1 / 2$, three to spin $3 / 2$, three to spin $5 / 2$ and two to spin $7 / 2$. The distributions significantly change as compared to those of the lower part of Fig. 1. Moreover, $\mathcal{P}_{\Lambda=10}\left(W_{i j}^{I, R}\right)$ (Eq. (7) ) (dashed lines) does not provide an optimal representation for these explicitly calculated distributions. For $E=20 \mathrm{MeV}$ particle energy (the upper part of Fig. 3), the best fit in terms of the formula (7) is obtained for $\Lambda_{\text {eff }}=3.1$ for the imaginary part and $\Lambda_{\text {eff }}=4.4$ for the real part of $W$. At $E=40 \mathrm{MeV}$ one obtains $\Lambda_{\text {eff }}=4.8$ and $\Lambda_{\text {eff }}=3.1$, correspondingly. This, first of all, indicates that effectively a smaller number of channels is involved what is caused by the broadening of the width distribution as a result of the non-equivalence of the channels 11 . Secondly, such effective characteristics depend on the energy of particle in the continuum, what in turn is natural in view of the dependences displayed in Fig. 2. It is also interesting to notice that $W_{i j}^{R}$ obeys functionally similar distribution as $W_{i j}^{I}$ although this does not result from Eq. (5) [8].

The fact that generically $\Lambda_{\text {eff }}$ is much smaller than the actual number of open physical channels can be anticipated from their obvious non-equivalence in majority of combinations as can be concluded from Fig. 2. The global distribution, especially in the tails, is dominated by stronger channels. Due to the separable form of $W$, which in terms of $\Lambda$ explicitly expresses its reduced dimensionality relative to $H_{Q Q}$, an interesting related effect in the eigenvalues of $H_{Q Q}^{e f f}$ may take place. For a sufficiently strong coupling to the continuum one may observe a segregation effect among the states, i.e., $\Lambda$ of them may separate from the remaining $M-\Lambda$ states [12]. This effect is especially transparent when looking at the structure of $W^{I}$. For the physical strength $V_{12}^{(0)}$ of the residual interaction in ${ }^{24} \mathrm{Mg}$ this effect is negligible, as shown in the upper panel of Fig. A. Only one state in this case separates from all others by acquiring a larger width. A magnification of the overall strength $V_{12}^{(0)}$ of the coupling to the continuum by a constant factor $f$ allows further states to consecutively separate. For $f=7$, all 10 states become unambigously separated as illustrated in the middle panel of Fig. 4. Their distance from the remaining, trapped states reflects approximately the order of their separation when $f$ is kept increasing. This nicely illustrates the degree of non-equivalence of the channels and the fact that $\Lambda_{\text {eff }} \approx 5$, as consistent with Fig. 3 at $E=40 \mathrm{MeV}$, is an appropriate representation for an effective number of relevant open channels. It needs also to be noticed that the segregation effect takes place also in the direction of the real energy axis, though in this sense only three states uniquely separate (again consis- 
tent with $\Lambda_{\text {eff }}=3.1$ of Fig. 3). This direction of the separation originates from the real part of $W$. Incorporating an equivalent multiplication factor into $W^{I}$ only, results in a picture as shown in the lower panel of Fig. 4. No separation in energy can now be observed anymore.

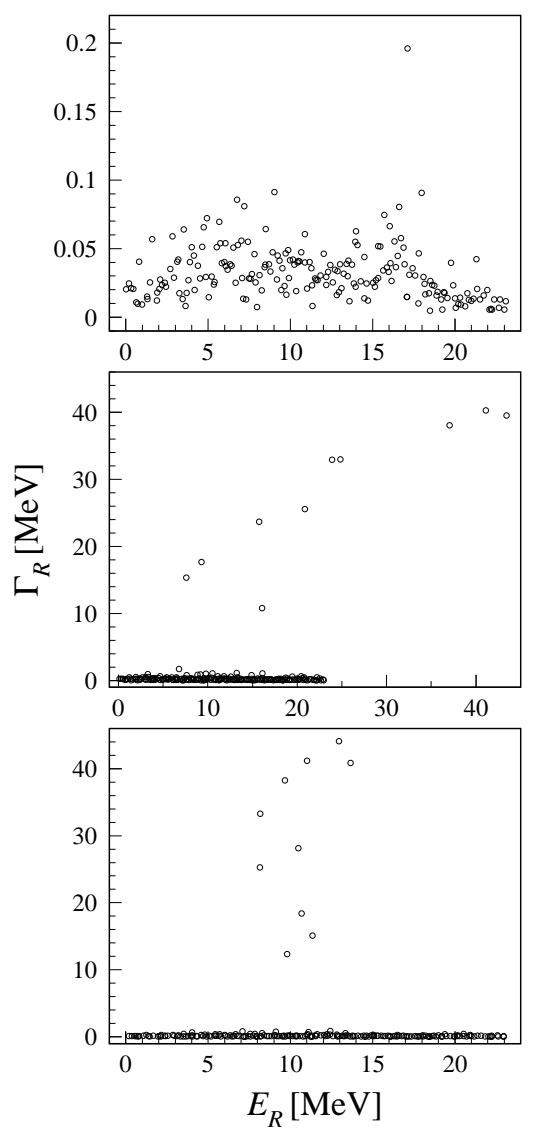

FIG. 4. 205 complex eigenvalues for 10 channels and energy of the particle in the continuum of $40 \mathrm{MeV}$ are presented as small circles with coordinates of $E_{R}$ and $\Gamma_{R}$. The upper part represents those for the original residual interaction between $Q$ and $P$ subspaces. The middle one is obtained for 7 times stronger interaction, and in the lower part this stronger force is applied to $W^{I}$ only.

In summary, the present study indicates that certain characteristics of the statistical description of nuclear coupling to the continuum, like the distribution of coupling matrix elements for one channel continuum, do indeed apply when the non-generic edge effects are removed. On the other hand, in realistic SMEC calculations we find the generic nonequivalence of channels which contradicts the orthogonal invariance arguments and results in strong reduction of the number of effectively involved channels. The quantitative identification and understanding of this effect may turn out to be helpful in postulating improved scattering ensembles which automatically account for this effect, similarly as various versions of the random matrix ensembles invented [1, 13, 14] in the context of bound states. Up to now the statistical models ignore the real part of the matrix connecting the bound states to the scattering states. The real part of $H_{Q Q}^{e f f}$ is likely to be dominated by $H_{Q Q}$, therefore, this, in many cases, may be not a bad approximation. Keeping in mind a relatively strong energy dependence of $W^{R}$ (see Fig. 2) this however may not be true in some cases, especially, because the segregation of states in energy (along the real axis) originates from this part. Interestingly, $W^{R}$ is found to obey similar statistical characteristics as $W^{I}$. This does not however yet mean that the two parts of $W$ can simply be drawn as independent ensembles. In fact, the individual matrix elements $W_{i j}^{I}$ and $W_{i j}^{R}$ are often strongly correlated and the degree of correlation depends on energy of the particle in the continuum. A more detailed account of such correlations will be presented elsewhere.

We thank K. Bennaceur, E. Caurier, F. Nowacki and M. Wójcik for useful discussions. This work was partly supported by KBN Grant No. 2 P03B 09716 and by the Grant No. 76044 of the French-Polish Cooperation.

[1] T.A. Brody, J. Flores, J.B. French, P.A. Mello, A. Panday, and S.S.M. Wong, Rev. Mod. Phys. 53, 385 (1981).

[2] V. Zelevinsky, B.A. Brown, N. Frazier, and M. Horoi, Phys. Rep. 276, 85 (1996).

[3] S. Drożdż, S. Nishizaki, J. Speth, and M. Wójcik, Phys. Rev. E 57, 4016 (1998).

[4] V.V. Sokolov, and V.G. Zelevinsky, Nucl. Phys. A 504, 562 (1989).

[5] S. Drożdż, A. Trellakis, and J. Wambach, Phys. Rev. Lett. 76, 4891 (1996).

[6] K. Bennaceur, F. Nowacki, J. Okołowicz, and M. Płoszajczak, Nucl. Phys. A 651, 289 (1999); nuclth/9909050.

[7] H.W. Barz, I. Rotter, and J. Höhn, Nucl. Phys. A 275, 111 (1977).

[8] It should be noted that the real part of Eq. (6) cannot be written in the dyadic product form.

[9] R. Blümel and U. Smilansky, Phys. Rev. Lett. 60, 477 (1988);

S. Drożdż, J. Okołowicz, and T. Srokowski, Phys. Rev. E 48, 4851 (1993).

[10] B.A. Brown and B.H. Wildenthal, Annu. Rev. Nucl. Part. Sci. 38, 191 (1988).

[11] E. Persson, T. Gorin and I. Rotter, Phys. Rev. E 54, 3339 (1996) and 58, 1334 (1998); see also V.A. Mandelshtam and H.S. Taylor, J. Chem. Soc. Faraday Trans. 93, 847 (1997).

[12] P. Kleinwächter and I. Rotter, Phys. Rev. C 32, 1742 (1985); W. Iskra, M. Müller, and I. Rotter, J. Phys. G 19, 2045 (1993); 20, 775 (1994).

[13] S.S.M. Wong, and J.B. French, Nucl. Phys. A 198, 188 (1972).

[14] C.W. Johnson, G.F. Bertsch, and D.J. Dean, Phys. Rev. Lett. 80, 2749 (1998). 